Article

\title{
State Feedback Regulation Problem to the Reaction-Diffusion Equation ${ }^{\dagger}$
}

\author{
Francisco Jurado *(i) and Andrés A. Ramírez \\ División de Estudios de Posgrado e Investigación, Tecnológico Nacional de México/I.T. La Laguna, Revolución \\ Blvd. and Instituto Tecnológico de La Laguna Av., Torreón 27000, Mexico; \\ m.aaramirezv@correo.itlalaguna.edu.mx \\ * Correspondence: fjurado@itlalaguna.edu.mx \\ $+\quad$ This paper is an extended version of our paper published in the Proceedings of the 2019 16th International \\ Conference on Electrical Engineering, Computing Science and Automatic Control (CCE), Mexico City, \\ Mexico, 11-13 September 2019.
}

Received: 13 September 2020; Accepted: 3 November 2020; Published: 6 November 2020

check for updates

\begin{abstract}
In this work, we explore the state feedback regulator problem (SFRP) in order to achieve the goal for trajectory tracking with harmonic disturbance rejection to one-dimensional (1-D) reaction-diffusion (R-D) equation, namely, a partial differential equation of parabolic type, while taking into account bounded input, output, and disturbance operators, a finite-dimensional exosystem (exogenous system), and the state of the exosystem as the state to the feedback law. As is well-known, the SFRP can be solved only if the so-called Francis (regulator) equations have solution. In our work, we try with the solution of the Francis equations from the 1-D R-D equation following given criteria to the eigenvalues from the exosystem and transfer function of the system, but the state operator is here defined in terms of the Sturm-Liouville differential operator (SLDO). Within this framework, the SFRP is then solved for the 1-D R-D equation. The numerical simulation results validate the performance of the regulator.
\end{abstract}

Keywords: applied mathematics; computational methods; exogenous system; reaction-diffusion equation; regulator problem; Sturm-Liouville differential operator; tracking

\section{Introduction}

Some physical quantities from applications involving the diffusion and structural vibrations depend on both position and time. Systems whose dynamic evolves in an infinite-dimensional Hilbert space are modeled by partial differential equations (PDEs). Such systems are called infinite-dimensional systems. Because these systems reflect the spatial distribution of a physical quantity, these systems are called distributed parameter systems (DPSs). The aim when designing the controller for infinite-dimensional systems is that the control system is stable and robust in the presence of parametric uncertainties and external perturbations.

The design of classical controllers is commonly based on the transfer function of the system. Infinite-dimensional systems have transfer function. Unlike transfer functions from finite-dimensional systems, transfer functions of infinite-dimensional systems are not rational functions. If the transfer function from an infinite-dimensional system is provided, then the controller can be directly designed. A drawback from this last approach is that the controller will be infinite-dimensional and it may be approximated by a finite-dimensional system. For some practical applications, it could be that a transfer function is not available. Subsequently, a finite-dimensional model of the system must be drawn from which the controller design may be based. This latter approach is the most used methodology to design controllers of systems modeled by PDEs [1]. 
The main aim in the regulator problem is the design of a feedback law, such that the output of the system tracks a reference signal in presence of a external disturbance, where the reference signal and external disturbances are generated through an exosystem (also called exogenous system). The state feedback regulation problem (SFRP) and error feedback regulation problem (EFRP) are two approaches that may be used to solve the output regulation problem [2]. The regulator theory has a long history and has been used in both finite- and infinite-dimensional cases. In this work, we try with the SFRP under the assumption that the whole state from the exosystem is available to the controller.

In our work, the employed methodology follows the work for linear finite-dimensional systems [3-6]. In [5], the solvability for the multivariable linear regulator problem, which tries with the solvability for a pair of linear matrix equations, namely, the Francis (regulator) equations, was shown. Necessary and sufficient conditions for the solvability of the so-called Hautus equations were given in [7]. In [8] the Hautus conditions were stated, for finite-dimensional linear systems, claiming that any eigenvalue from the exosystem is not an invariant zero of the control system. In [3] the regulator problem was extended to finite-dimensional nonlinear systems where necessary and sufficient conditions, about the solvability of a pair of nonlinear regulator equations, are given to the solvability of the problem.

The regulator problem for linear finite-dimensional systems was extended to linear DPSs [9-13]. Control systems governed by a discrete spectral operator are presented in [11,12], where the so-called state operator comply with the property of spectrum decomposition [14,15] from which a controllability condition is determined by the spectrum, implying the stabilizability of the control system through a finite-dimensional controller. In [13], the geometric regulation approach was extended to linear DPSs with bounded input and output operators where both reference and disturbance signals considered in $[11,12]$ are assumed as generated by a finite-dimensional exosystem. Additionally, simple criteria for the solvability of the Francis (regulator) equations were provided. Moreover, the regulator problem to set-point control with harmonic disturbance, such disturbance acting over a small spatial interval, for a one-dimensional (1-D) heat equation, the regulator problem for harmonic tracking of a damped wave equation, when considering full state feedback for both cases, as well as the regulator problem for harmonic tracking of a 1-D heat equation, but under the error feedback approach, these latter without considering disturbances, were solved. Neumann boundary conditions were considered to the cases mentioned above about the regulator problem for a 1-D heat equation. The case for regulation of linear systems with bounded input, output and disturbance operators, this last disturbance operator entering across the entire interval, was considered in [16] for a 1-D heat equation with Dirichlet condition at one boundary and Neumann condition at the other boundary. In [17] key results from [13], regarding the state feedback regulator problem, were extended to systems with unbounded control and observation operators. The regulator theory developed in such a work was applied to a one-dimensional (1-D) heat equation, a heat equation on a two-dimensional (2-D) unit rectangle, and on a damped Rayleigh beam.

Comprehensive reviews regarding contributions made to generalize the regulator problem to infinite-dimensional systems can be found in $[17,18]$. The output regulation problem for DPSs has been extensively studied for different classes of PDEs systems; for example, parabolic and hyperbolic PDEs [18-20], linearized Stokes flow, and steady-state incompressible Navier-Stokes fluid flow equations with spatially varying parameters [16], coupled wave equation [16], wave equations [21,22], beam and plate equations $[16,17,23,24]$, and partial integro-differential equations [25]. In most of the works, the controller is done based on output feedback; these include system stabilization and availability of the state of the exosystem for feedback is not required.

Reaction-diffusion (R-D) equations have been playing an important role in applied mathematics. Dynamics from combustion processes, superconductivity in liquids, signal transmission across neurons, population growth, mass balance and mass transport, chemical kinetics, chemotaxis, metabolic processes in living cells, and the spread of diseases and viruses can be analyzed and simulated by R-D models [26-34]. In [35], boundary control to the R-D equation via the backstepping method was proposed. The main idea of using the backstepping method was about the use of a Volterra integral transformation along with feedback control that made that the R-D system behaves as 
the heat equation; this latter as an exponentially stable target system. To the best of our knowledge, there is no work regarding the solution of the SFRP for harmonic tracking with harmonic disturbance rejection to the 1-D R-D equation.

In this work, although it could be argued that the 1-D R-D equation is be among the simplest PDEs systems, our application is concerned with the SFRP to the design of a harmonic tracking regulator, but, in contrast with our previous work recently reported in [36], with harmonic disturbance rejection for this kind of system. It is well known that the SFRP is solved only if the Francis (regulator) equations have a solution. In our work, the main contribution is that the state operator to the Francis equations, corresponding to the 1-D R-D equation, here is defined in terms of the Sturm-Liouville differential operator (SLDO) to then solve them.

The manuscript is organized, as follows. In Section 1, the SFRP, modeling of DPS, control of PDEs, and applications of the R-D equation are summarized; the problem statement is formulated in Section 2; the regulator design is detailed at Section 3; the simulation results are included in Section 4; and, the conclusion is drawn at the end.

\section{Problem Statement}

\subsection{Sturm-Liouville Boundary Value Problem}

Let us consider a differential equation of the form

$$
\left(p(x) y^{\prime}\right)^{\prime}-q(x) y+\lambda r(x) y=0, \quad x \in(0,1),
$$

with symmetric boundary conditions, also known as Sturm-Liouville boundary conditions,

$$
\alpha_{1} y(0)+\alpha_{2} y^{\prime}(0)=0, \quad \beta_{1} y(1)+\beta_{2} y^{\prime}(1)=0,
$$

at the endpoints. The Sturm-Liouville Equation (1) can be rewritten as

$$
\sum \frac{\partial}{\partial x_{i}}\left(p(x) \frac{\partial y}{\partial x_{i}}\right)-q(x) y+\lambda r(x) y=0
$$

or, equivalently,

$$
p(x) \Delta y-q(x) y+\lambda r(x) y=0,
$$

with $\Delta$ the Laplacian operator [26,37], assuming that $p(x)$ is a $\mathcal{C}^{1}$ function, $q(x)$ and $r(x)$ are continuous functions, $p(x)>0$, and $r(x)>0$ [38]. In this case, the boundary value problem is said to be regular. It should be noticed that these latter conditions are satisfied in many problems in mathematical physics.

$$
L=-\frac{d}{d x} p(x) \frac{d}{d x}+q(x)
$$

is known as the SLDO [39].

Let us consider the linear homogeneous differential operator

$$
L[y]=-\left(p(x) y^{\prime}\right)^{\prime}+q(x) y .
$$

Accordingly, the Equation (1) can be written as

$$
L[y]=\lambda r(x) y .
$$

Each one of the boundary conditions (2) involves only one of the boundary points, and that is why are said to be separated. In order to impose any restriction on $y$, at least one of the parameters $\alpha_{1}$ and $\alpha_{2}$ must be different from zero to the first boundary condition. Alike, at least one of the parameters 
$\beta_{1}$ and $\beta_{2}$ must differ from zero [40]. The boundary value problem consisting of (1) and (2) is called the Sturm-Liouville boundary value problem (SLBVP) [41].

Modeling that involves ordinary differential equations (ODEs) usually leads to initial value problems or boundary value problems, these latter written in the form of the SLBVPs, which are eigenvalue problems that involve a parameter $\lambda$ related to frequencies, energies, or other physical quantities [42]. In fact, the next theorem summarizes the way about how to convert a linear second order differential equation $A(x) y^{\prime \prime}+B(x) y^{\prime}+C(x) y$ into one of the form $(1 / r(x))\left(\left(p(x) y^{\prime}\right)^{\prime}+q(x) y\right)$. If $p(x), q(x)$, and $r(x)$ are given, then $A(x)=p(x) / r(x), B(x)=p(x)^{\prime} / r(x)$, and $C(x)=q(x) / r(x)$.

Theorem 1. [43] Suppose that $A(x)>0, B(x)$ and $C(x)$ are analytic real-valued functions in the (finite or infinite) interval $a<x<b$, then exist functions $p(x)>0, q(x)$, and $r(x)>0$ likewise analytic and real valued in $a<x<b$ and

$$
A(x) y^{\prime \prime}+B(x) y^{\prime}+C(x) y=\frac{1}{r(x)}\left[\left(p(x) y^{\prime}\right)^{\prime}+q(x) y\right]
$$

identically in $y$.

Proof. See [43].

The expression on the right from this last equation is referred as the Sturmian form of the differential equation, also called self-adjoint form. Solutions of SLBVPs, called eigenfunctions, have many general properties in common, as the orthogonality property, useful in eigenfunctions expansions in terms of Fourier series, Legendre polinomials, Bessel functions, and other eigenfunctions [41,44].

\subsection{Abstract Control System Model}

Let us consider an abstract distributed parameters control system in the Hilbert space $\mathcal{Z}$ in the form

$$
\begin{aligned}
z_{t} & =\mathcal{A} z(t)+B_{\text {in }} u(t)+B_{d} d(t), \\
z(0) & =z_{0}, \quad z_{0} \in \mathcal{Z}, \\
y(t) & =C z(t),
\end{aligned}
$$

where the state operator $\mathcal{A}$ refers to an unbounded densely defined operator, $z(t)$ stands for the state of the system, $z_{0}$ is for the initial condition, $z_{t}$ is the derivative of $z(t)$ with respect to time, $u(t) \in \mathcal{U}$ is the control input, and $y(t) \in \mathcal{Y}$ is the measured output. $\mathcal{U}, \mathcal{Y}$ are Hilbert spaces of either finite or infinite dimension. $B_{\text {in }}$ is an input operator, $B_{d} \in \mathcal{L}(\mathcal{U}, \mathcal{Z})$ is a disturbance operator, $d(t)$ refers to the disturbance, and $C$ is an output operator.

The operator $\mathcal{A}$ is given in terms of a linear elliptic partial differential operator $L$ in the Hilbert space $\mathcal{Z}=L^{2}(\Omega)$ of infinite dimension with bounded domain $\Omega \in \mathbb{R}^{n}$ with piecewise smooth boundary. To the second order case,

$$
L=\sum_{i, j=1}^{n} \frac{\partial}{\partial x_{j}}\left(a_{i j}(x) \frac{\partial}{\partial x_{i}}\right)-a(x)
$$

where $a(x) \geq 0$ for all $x \in \Omega, a_{i j} \in \mathcal{C}^{\infty}(\bar{\Omega}), a_{i j}(x)=a_{j i}(x)$, and $\bar{\Omega}$ is the closure of $\Omega$. If there exist constants $0<c_{1}<c_{2}<\infty$, by uniform ellipticity it means that

$$
c_{1}|\xi|^{2} \leq \sum_{i, j=1}^{n} a_{i j}(x) \xi_{i} \xi_{j} \leq c_{2}|\xi|^{2}
$$

for all $\xi \in \mathbb{R}^{n}$ and $x \in \bar{\Omega}$, where $|\cdot|$ represents the Euclidean norm in $\mathbb{R}^{n}$. 
In our work, from (5) and (11), the state operator is defined here as given in the form of the SLDO, namely,

$$
\mathcal{A}=A+\lambda,
$$

with

$$
A=d^{2} / d x^{2} .
$$

The output operator $C \in \mathcal{L}(\mathcal{Z}, \mathcal{Y})$ is a set of bounded operators $C_{i}$ given by

$$
y_{i}(t)=C_{i} z=\frac{1}{\left|\Omega_{i}\right|} \int_{\Omega_{i}} z(x, t) d x,
$$

for some $\Omega_{j}$ of the domain $\Omega$, with Lebesgue measure

$$
\left|\Omega_{i}\right|=\int_{\Omega_{i}} d x>0 .
$$

In the same setting,

$$
y_{i}(t)=C_{i} z=\left\langle z, \Psi_{i}\right\rangle=\int_{\Omega} z(x, t) \Psi_{i}(x) d x
$$

with

$$
\Psi_{i}(x)=\frac{1}{\left|\Omega_{i}\right|} \mathbf{1}_{\Omega_{i}}(x) \in L^{2}(\Omega)
$$

and indicator function

$$
\mathbf{1}_{\Omega_{i}}(x)= \begin{cases}1, & x \in \Omega_{i} \\ 0, & x \notin \Omega_{i} .\end{cases}
$$

Accordingly,

$$
y=C z=\left[\begin{array}{llll}
C_{1}(z) & C_{2}(z) & \cdots & C_{n_{c}}(z)
\end{array}\right]^{T} .
$$

2.3. Exogenous System (Exosystem)

Let us consider a finite-dimensional exosystem

$$
\begin{aligned}
\frac{d w(t)}{d t} & =S w(t), \\
y_{r}(t) & =Q w(t), \\
d(t) & =P w(t), \\
w(0) & =w_{0},
\end{aligned}
$$

which generates both reference output $y_{r}(t)$ and disturbance $d(t), \mathcal{W}$ is the state space of the exosystem, $S \in \mathcal{L}(\mathcal{W}), Q \in \mathcal{L}(\mathcal{W}, \mathcal{Y})$, and $P \in \mathcal{L}(\mathcal{W}, \mathcal{Z})$.

Let us consider the system given by

$$
\begin{aligned}
z_{t}(x, t) & =\mathcal{A} z(x, t), \\
z(x, 0) & =z_{0}(x) .
\end{aligned}
$$

If there exist positive constants $M$ and $\alpha$, such that

$$
\|z(\cdot, t)\|=\left\|e^{\mathcal{A} t} z_{0}\right\| \leq M e^{-\alpha t}\left\|z_{0}\right\| \quad \forall t \geq 0,
$$


with $z_{0} \in \mathcal{Z}$, then the system (20) and (21) is exponentially stable. From the above, it is said that the state operator $\mathcal{A}$ generates an exponentially stable $C_{0}$ semigroup in $\mathcal{Z}$ [45], i.e., $\mathcal{A}$ is stable. Accordingly, here it is assumed that the uncontrolled system (20) and (21), i.e., to the case when $u=0$ and $d=0$, is exponentially stable. Additionally, it is assumed that the exogenous system (16)-(19) is neutrally stable. To the linear case, in the Lyapunov sense, this is equivalent to the origin being stable implying that $\sigma(S) \subset i \mathbb{R}$ and $S$ has no nontrivial Jordan blocks. $\rho(T)$ refers to the resolvent set of an operator $T$ and $\sigma(T)$ refers to the spectrum of $T$.

Let us consider the error signal

$$
\begin{aligned}
e(t) & =y(t)-y_{r}(t) \\
& =C z(t)-Q w(t) .
\end{aligned}
$$

The main task for the regulator is to force the output of the system to track a reference signal in presence of a disturbance $d(t)$, i.e., $e(t) \rightarrow 0$ as $t \rightarrow \infty$. Accordingly, the problem is stated as follows.

Problem 1. State Feedback Regulator Problem (SFRP).

The SFRP consists in to find a control law

$$
u(t)=\Gamma w(t)
$$

in function of the state of the exosystem with $\Gamma \in \mathcal{L}(\mathcal{W}, \mathcal{U})$, such that for the system

$$
\begin{aligned}
\frac{d z(t)}{d t} & =\mathcal{A} z(t)+\left(B_{d} P+B_{i n} \Gamma\right) w(t), \\
\frac{d w(t)}{d t} & =S w(t),
\end{aligned}
$$

which corresponds to the interconnection of (8)-(10) with (16)-(19), the error norm

$$
\begin{aligned}
\|e(t)\| & =\left\|y(t)-y_{r}(t)\right\|, \\
& =\|C z(t)-Q w(t)\|,
\end{aligned}
$$

satisfies

$$
\|e(t)\| \rightarrow 0 \text { as } t \rightarrow \infty,
$$

for any $z_{0} \in \mathcal{Z}$ and $w_{0} \in \mathcal{W}$.

Being $e(t)$ a finite dimension vector, then all $l_{p}$ norms in (30) are equivalent. Because exponential stability for the system (20) and (21) has been assumed, then a state feedback control law is not involved. In this work, our main focus is with tracking and harmonic disturbance rejection, so, the stabilization problem is out of scope of our proposal. Thus, the solvability of the SFRP is stated in the next theorem.

Theorem 2. If there exist mappings $\Pi \in \mathcal{L}(\mathcal{W}, \mathcal{Z})$ and $\Gamma \in \mathcal{L}(\mathcal{W}, \mathcal{U})$, with $\operatorname{rank}(\Pi) \subset D(\mathcal{A})$, satisfying the Francis (regulator) equations

$$
\begin{aligned}
& \Pi S=\mathcal{A} \Pi+B_{\text {in }} \Gamma+B_{d} P, \\
& C \Pi=Q,
\end{aligned}
$$

then the linear SFRP has solution. The feedback control law that solves the SFRP is given by

$$
u(t)=\Gamma w(t) .
$$


Proof. The proof can be carried out along the same lines as in [16].

From this last theorem, from the assumptions that $e^{\mathcal{A} t}$ is an exponentially stable semigroup and that the exosystem is neutrally stable, if (31) holds then $e(t) \rightarrow 0$ as $t \rightarrow \infty$ for all $z_{0} \in Z, w_{0} \in W$, if and only if $[C \Pi-Q]=0$.

\section{Regulator Design}

Let us consider the 1-D R-D equation that is given by

$$
\begin{array}{rlr}
z_{t}(x, t) & =\mathcal{D} z_{x x}(x, t)+\lambda z(x, t)+B_{i n} u+B_{d} d, \\
z(0, t) & =0, \quad \quad \quad \quad \text { (Dirichlet BC) } \\
z_{x}(1, t) & =0, \quad \text { (Neumann BC) } \\
z(x, 0) & =\phi(x), & \\
y(t) & =C z(t), &
\end{array}
$$

where $\mathcal{D} z_{x x}(x, t)$ is referred as the diffusion term, with diffusion coefficient (constant) $\mathcal{D}>0$, and $\lambda z(x, t)$ is the reaction (source) term, with $\lambda$ an arbitrary constant. Refer to [37] to have an idea about typical values that $\mathcal{D}$ may take when trying with heat conduction or molecular diffusion.

The system (34)-(38) is formulated in the form (8)-(10) in the Hilbert state space $\mathcal{Z}=L^{2}(0,1)$. $z_{x}$ refers to the partial derivative with respect to space and $z_{x x}$ refers to the second partial derivative with respect to space also. The maximal elliptic operator is given by $L=d^{2} / d x^{2}$ in (11) with domain $D(L)=H^{2}(0,1)$, the Sobolev space of functions $\zeta \in \mathcal{Z}$ with $d \zeta / d x$ both continuous on $(0,1)$ and $d^{2} \zeta / d x^{2} \in \mathcal{Z}$. The state operator (12) is a self-adjoint operator in $\mathcal{Z}$, i.e.,

$$
\mathcal{A}[\zeta]=\zeta^{\prime \prime}+\lambda \zeta
$$

where

$$
D(\mathcal{A})=\left\{\zeta \in H^{2}(0,1): \zeta(0)=0, \zeta^{\prime}(1)=0\right\} \subset \mathcal{Z} .
$$

The spectrum of $\mathcal{A}$ denoted by

$$
\sigma(\mathcal{A})=\left\{\lambda_{k}\right\}_{k=0}^{\infty}
$$

where $\lambda_{k}=-\mu_{k}^{2}$ with $\mu_{k}=\left(k-\frac{1}{2}\right) \pi$, is purely discrete with a corresponding set of orthonormal eigenvectors

$$
\zeta_{k}(x)=\sqrt{2} \sin \left(\mu_{k} x\right), \quad k=1,2, \ldots
$$

The operator $\mathcal{A}$ is assumed as an infinitesimal generator of an exponentially stable $C_{0}$ semigroup in $\mathcal{Z}$ in terms of the eigenfunction expansion

$$
e^{\mathcal{A} t} \zeta=\sum_{j=0}^{\infty} e^{\lambda_{j} t}\left\langle\zeta, \zeta_{j}\right\rangle \zeta_{j} .
$$

It should be noticed that the system under consideration is a single-input/single-output system with scalar input and output $B_{i n}$ and $C$, respectively, and $B_{d}$ is for a bounded disturbance. Additionally, the input, output, and disturbance operators are bounded operators acting in the interior of the domain.

The input to the system is spatially uniform over a small interval about a fixed point $x_{i n}=x_{0} \in$ $(0,1)$, where $B_{\text {in }} u=b(x) u$ with

$$
b(x)=\frac{1}{2 v_{0}} \mathbf{1}_{\left[x_{0}-v_{0}, x_{0}+v_{0}\right]}(x)
$$


and

$$
1_{[a, b]}(x)= \begin{cases}1, & x \in[a, b] \\ 0, & x \notin[a, b] .\end{cases}
$$

The input operators $B_{i n}$ and $B_{d}$ are given as

$$
B_{\text {in }} u(t)=\sum_{j=1}^{n_{\text {in }}} B_{\text {in }}^{j} u_{j}(t), \quad B_{d} d(t)=\sum_{j=1}^{n_{d}} B_{d}^{j} d_{j}(t),
$$

where $u_{j}(t)$ and $d_{j}(t)$ are scalar control inputs and disturbances, respectively. $B_{i n}^{j}$ and $B_{d}^{j}(x)$ are characteristic functions of a bounded subset of $\Omega$, namely

$$
B_{i n}^{j}(x)=\frac{1}{\left|\Omega_{j}\right|} \mathbf{1}_{\Omega_{j}}(x) .
$$

To guarantee that $B_{i n}^{j} \in \mathcal{Z}$, here it is assumed that $\left|\Omega_{j}\right|>0$.

The output is the average transport reaction over a small interval about a point $x_{\text {out }}=x_{1} \in$ $(0,1)$, i.e.,

$$
C \phi=\int_{0}^{1} c(x) \phi(x) d x
$$

with

$$
c(x)=\frac{1}{2 v_{1}} \mathbf{1}_{\left[x_{1}-v_{1}, x_{1}+v_{1}\right]}(x) .
$$

Because $C \zeta=\langle\zeta, c\rangle, C$ is a bounded linear observation functional on $\mathcal{Z}$.

In our work, $d(t)=A_{d} \sin (\beta t) \in \mathbb{R}$ entering across the whole interval is considered, so $B_{d}=1$. In our proposal, the Francis Equations (31) and (32) take the form

$$
\begin{aligned}
& \Pi S w=\mathcal{A} \Pi w+B_{i n} \Gamma w+B_{d} P w \\
& C \Pi w=Q w=w_{1},
\end{aligned}
$$

with

$$
\begin{gathered}
\Pi=\left[\begin{array}{llll}
\Pi_{1} & \Pi_{2} & \Pi_{3} & \Pi_{4}
\end{array}\right], \quad w(t)=\left[\begin{array}{l}
w_{1} \\
w_{2} \\
w_{3} \\
w_{4}
\end{array}\right]=\left[\begin{array}{l}
A_{r} \sin (\alpha t) \\
A_{r} \cos (\alpha t) \\
A_{d} \sin (\beta t) \\
A_{d} \cos (\beta t)
\end{array}\right], \\
S=\left[\begin{array}{cccc}
0 & \alpha & 0 & 0 \\
-\alpha & 0 & 0 & 0 \\
0 & 0 & 0 & \beta \\
0 & 0 & -\beta & 0
\end{array}\right], \quad Q=\left[\begin{array}{llll}
1 & 0 & 0 & 0
\end{array}\right], \quad P=\left[\begin{array}{llll}
0 & 0 & 1 & 0
\end{array}\right] .
\end{gathered}
$$

Because of the block diagonal structure of $S$, the Francis equations can be decoupled into two separate parts, the first one to work with the harmonic tracking task and the last one to work with the rejection of a harmonic disturbance. 


\subsection{Harmonic Tracking}

Accordignly, along the same lines from [16], for the first part on which it is desirable to track a harmonic signal $y_{r}(t)=A_{r} \sin (\alpha t)$ consider an exosystem given as

$$
w_{t}=S w, \quad w(0)=\left[\begin{array}{c}
0 \\
A_{r}
\end{array}\right], \quad S=\left[\begin{array}{cc}
0 & \alpha \\
-\alpha & 0
\end{array}\right],
$$

with solution

$$
w(t)=\left[\begin{array}{l}
A_{r} \sin (\alpha t) \\
A_{r} \cos (\alpha t)
\end{array}\right]
$$

Taking $P=\left[\begin{array}{ll}0 & 0\end{array}\right]$ and $Q=\left[\begin{array}{ll}1 & 0\end{array}\right]$ then

$$
y_{r}(t)=Q w=A_{r} \sin (\alpha t) .
$$

In this case $\mathcal{W}=\mathbb{R}^{2}$, so $\Pi=\left[\begin{array}{ll}\Pi_{1} & \Pi_{2}\end{array}\right]$ and $\Gamma=\left[\begin{array}{ll}\Gamma_{1} & \Gamma_{2}\end{array}\right] \in \mathbb{R}^{2}$ with $\Pi_{j} \in \mathcal{Z}$.

The Francis equations applied to the vector $w=\left[\begin{array}{ll}w_{1} & w_{2}\end{array}\right]^{T} \in \mathcal{W}$ give the following system

$$
\begin{aligned}
& \Pi S w=\mathcal{A} \Pi w+B_{i n} \Gamma w, \\
& C \Pi w=Q w .
\end{aligned}
$$

From these last equations, the regulator equation on the left can be written as

$$
\alpha \Pi_{1} w_{2}-\alpha \Pi_{2} w_{1}=\mathcal{A} \Pi_{1} w_{1}+\mathcal{A} \Pi_{2} w_{2}+B_{i n} \Gamma_{1} w_{1}+B_{\text {in }} \Gamma_{2} w_{2} .
$$

Because (46) must be fulfilled for all $w$, let us consider first the case $w_{1}=1$ and $w_{2}=0$ and then $w_{1}=0$ and $w_{2}=1$ yielding

$$
\begin{aligned}
-\alpha \Pi_{2}-\mathcal{A} \Pi_{1} & =B_{\text {in }} \Gamma_{1}, \\
\alpha \Pi_{1}-\mathcal{A} \Pi_{2} & =B_{\text {in }} \Gamma_{2} .
\end{aligned}
$$

Remarking that the exogenous system (16)-(19) is neutrally stable, multiplying (48) by $i=\sqrt{-1}$ to then adding the result to (47) it yields

$$
(i \alpha I-\mathcal{A}) \Pi_{1}+i(i \alpha I-\mathcal{A}) \Pi_{2}=B_{i n} i \Gamma_{2}+B_{i n} \Gamma_{1} .
$$

Because $i \alpha \notin \rho(\mathcal{A})$, multiplying from the left by $(i \alpha I-\mathcal{A})^{-1}$ both sides of (49) results

$$
\Pi_{1}+i \Pi_{2}=(i \alpha I-\mathcal{A})^{-1} B_{i n}\left(\Gamma_{1}+i \Gamma_{2}\right) .
$$

Applying $C$ to both sides of (50) and recalling that $C \Pi w=Q w$, with $C \Pi_{1}=1$ and $C \Pi_{2}=0$, then $Q=\left[\begin{array}{ll}1 & 0\end{array}\right]$. Consequently,

$$
1=C(i \alpha I-\mathcal{A})^{-1} B_{i n}\left(i \Gamma_{2}+\Gamma_{1}\right)=G(i \alpha)\left(\Gamma_{1}+i \Gamma_{2}\right) .
$$

Rewriting (51) in terms of $G(i \alpha)=\operatorname{Re}(G(i \alpha))+i \operatorname{Im}(G(i \alpha))$, it yields

$$
1=(\operatorname{Re}(G(i \alpha))+i \operatorname{Im}(G(i \alpha)))\left(\Gamma_{1}+i \Gamma_{2}\right)
$$


From the above, matching real and imaginary parts,

$$
\begin{aligned}
& 1=\left(\operatorname{Re}(G(i \alpha)) \Gamma_{1}-\operatorname{Im}(G(i \alpha)) \Gamma_{2},\right. \\
& 0=\left(\operatorname{Im}(G(i \alpha)) \Gamma_{1}+\operatorname{Re}(G(i \alpha)) \Gamma_{2} .\right.
\end{aligned}
$$

Hence,

$$
\Gamma_{1}=\frac{\operatorname{Re}(G(i \alpha))}{|G(i \alpha)|^{2}}, \quad \Gamma_{2}=-\frac{\operatorname{Im}(G(i \alpha))}{|G(i \alpha)|^{2}} .
$$

Accordingly, from the fact that

$$
G(i \alpha)^{-1}=\frac{1}{G(i \alpha)}=\frac{\overline{G(i \alpha)}}{|G(i \alpha)|^{2}}=\frac{\operatorname{Re}(G(i \alpha))-i \operatorname{Im}(G(i \alpha))}{|G(i \alpha)|^{2}}
$$

the desired control is given by

$$
\Gamma=\left[\begin{array}{ll}
\Gamma_{1} & \Gamma_{2}
\end{array}\right]=\left[\operatorname{Re}\left(G(i \alpha)^{-1}\right) \quad \operatorname{Im}\left(G(i \alpha)^{-1}\right)\right] .
$$

Here, it has been assumed that the system is real, i.e., $G(\bar{s})=\overline{G(s)}$ for all $s \notin \rho(\mathcal{A})$. It should be noticed that $G(i \alpha) \neq 0$ for solvability.

\subsection{Harmonic Disturbance Rejection}

Now, for the last part where is desirable to reject a sinusoidal disturbance such as $d(t)=$ $A_{d} \sin (\beta t)$, let us chose an exosystem that is governed by the harmonic oscillator

$$
w_{t}=S w, w(0)=\left[\begin{array}{c}
0 \\
A_{d}
\end{array}\right], S=\left[\begin{array}{cc}
0 & \beta \\
-\beta & 0
\end{array}\right],
$$

with solution

$$
w(t)=\left[\begin{array}{l}
A_{d} \sin (\beta t) \\
A_{d} \cos (\beta t)
\end{array}\right]
$$

Taking $Q=\left[\begin{array}{ll}0 & 0\end{array}\right]$ and $P=\left[\begin{array}{ll}1 & 0\end{array}\right]$, then

$$
y_{r}(t)=Q w=0
$$

Again, to this case $\mathcal{W}=\mathbb{R}^{2}$ so, we look for $\Pi=\left[\begin{array}{ll}\Pi_{3} & \Pi_{4}\end{array}\right]$, where $\Pi_{j} \in \mathcal{Z}$, and $\Gamma=\left[\begin{array}{ll}\Gamma_{3} & \Gamma_{4}\end{array}\right] \in \mathbb{R}^{2}$. The Francis equations that are applied to the vector $w=\left[\begin{array}{ll}w_{3} & w_{4}\end{array}\right]^{T} \in \mathcal{W}$ result in the system

$$
\begin{aligned}
& \Pi S w=\mathcal{A} \Pi w+B_{d} P w+B_{i n} \Gamma w \\
& C \Pi w=0 .
\end{aligned}
$$

Expanding the above matrix multiplications, the regulator equation from the left becomes

$$
\beta \Pi_{3} w_{4}-\beta \Pi_{4} w_{3}=\mathcal{A} \Pi_{3} w_{3}+\mathcal{A} \Pi_{4} w_{4}+B_{d} w_{3}+B_{i n} \Gamma_{3} w_{3}+B_{i n} \Gamma_{4} w_{4} .
$$


Because (55) has to hold for $w$, now consider the case for $w_{3}=1$ and $w_{4}=0$ to then consider that for $w_{3}=0$ and $w_{4}=1$ resulting

$$
\begin{aligned}
-\beta \Pi_{4}-\mathcal{A} \Pi_{3} & =B_{d}+B_{i n} \Gamma_{3} \\
\beta \Pi_{3}-\mathcal{A} \Pi_{4} & =B_{i n} \Gamma_{4} .
\end{aligned}
$$

By multiplying (57) by $i=\sqrt{-1}$ and adding the result to (56), we get

$$
(i \beta I-\mathcal{A}) \Pi_{3}+i(i \beta I-\mathcal{A}) \Pi_{4}=B_{d}+B_{i n} i \Gamma_{4}+B_{i n} \Gamma_{3} .
$$

Noting that $i \beta \notin \rho(\mathcal{A})$, applying $(i \beta I-\mathcal{A})^{-1}$ to both sides of $(58)$, we get

$$
\Pi_{3}+i \Pi_{4}=(i \beta I-\mathcal{A})^{-1} B_{d}+(i \beta I-\mathcal{A})^{-1} B_{\text {in }}\left(i \Gamma_{4}+\Gamma_{3}\right) .
$$

By multiplying both sides of (59) by $C$, from (54) implies

$$
C \Pi_{3}=0, \quad C \Pi_{4}=0,
$$

thus, we get

$$
\begin{aligned}
0 & =C(i \beta I-\mathcal{A})^{-1} B_{d}+C(i \beta I-\mathcal{A})^{-1} B_{i n}\left(i \Gamma_{4}+\Gamma_{3}\right) \\
& =G_{B_{d}}(i \beta)+G(i \beta)\left(i \Gamma_{4}+\Gamma_{3}\right)
\end{aligned}
$$

where we have used the notation

$$
G_{B_{d}}(s)=C(s I-\mathcal{A})^{-1} B_{d}
$$

Finally, solving for $\Gamma$ we have

$$
\begin{aligned}
& \Gamma_{3}=-\operatorname{Re}\left(G(i \beta)^{-1}\right) \operatorname{Re}\left(G_{B_{d}}(i \beta)\right)+\operatorname{Im}\left(G(i \beta)^{-1}\right) \operatorname{Im}\left(G B_{d}(i \beta)\right), \\
& \Gamma_{4}=-\operatorname{Re}\left(G(i \beta)^{-1}\right) \operatorname{Im}\left(G_{B_{d}}(i \beta)\right)-\operatorname{Im}\left(G(i \beta)^{-1}\right) \operatorname{Re}\left(G B_{d}(i \beta)\right),
\end{aligned}
$$

where

$$
\Gamma=\left[\begin{array}{ll}
\Gamma_{3} & \Gamma_{4}
\end{array}\right]
$$

Consequently, by combining (52) and (60) we get

$$
\Gamma=\left[\begin{array}{llll}
\Gamma_{1} & \Gamma_{2} & \Gamma_{3} & \Gamma_{4}
\end{array}\right]
$$

Although it could be argued that the 1-D R-D equation was so easy to find an explicit formula for the transfer function $G(s)=C(s I-\mathcal{A})^{-1} B_{i n}$, then there is no reason for which the state feedback regulator problem to this type of PDE had not been reported in the literature in spite of its importance to a myriad of applications.

\section{Simulation Results}

In our numerical simulation, we have set $A_{d}=2, A_{r}=2, \alpha=4, \beta=4, x_{0}=0.75, x_{1}=0.25$ and $v_{0}=v_{1}=0.25$. From Figures $1-3$, it can be seen that the controlled output $y(t)$ tracks the reference signal $y_{r}(t)$ from an initial condition $\varphi(x)=4 \cos (\pi x)$. Additionally, it can be seen that the error signal $e(t)$ tends to zero as time tends to infinity. The performance of the regulator is shown for different $\lambda$ values. The convergence of $y(t)$ to $y_{r}(t)$ is faster for small $\lambda$ values. From Figures $4-6$, the solution surface is shown for every $\lambda$ value. In mass balance the source term $\lambda z$ can be interpreted 
as the rate of production (destruction) of species per unit volume. From (34), the sign from the diffusion term agrees with the observation that mass flows from high to low mass concentration. In general, pressure gradients, temperature, and external forces affect the mass flux but their effects can be neglected and take the diffusion coefficient $\mathcal{D}>0$ to be constant. In fact, the units may be chosen in a convenient way in order to make $\mathcal{D}=1$. In our work, the diffusion coefficient is set to the unit value.

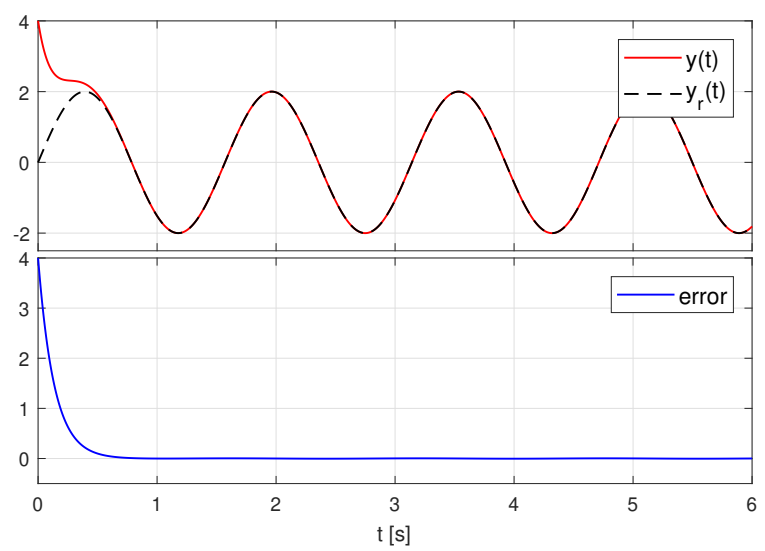

Figure 1. Performance of the regulator for $\lambda=2.5$ with $\Gamma_{1}=1.8424, \Gamma_{2}=1, \Gamma_{3}=-0.5$, and $\Gamma_{4}=0$.

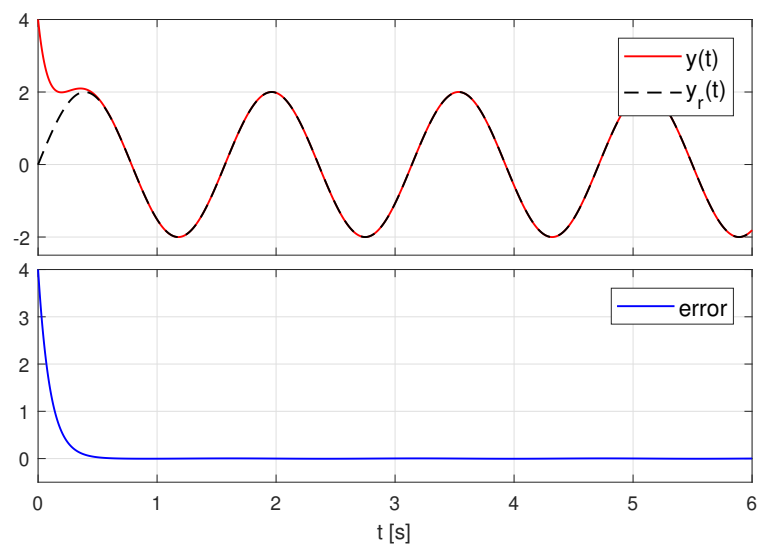

Figure 2. Performance of the regulator for $\lambda=0$, i.e., to the case for which the R-D equation is reduced to the Fick's second law of diffusion (also called simply as diffusion equation), for whose case $\Gamma_{1}=2.4674$, $\Gamma_{2}=1, \Gamma_{3}=-0.5$, and $\Gamma_{4}=0$.

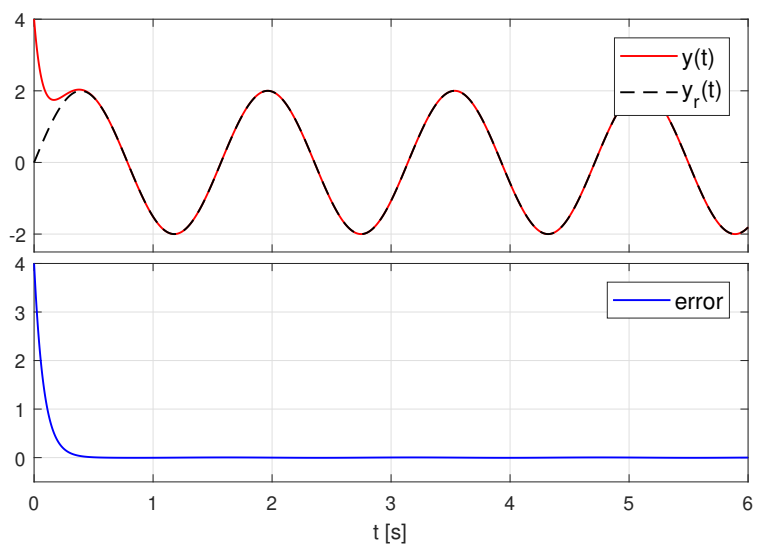

Figure 3. Performance of the regulator for $\lambda=-2.5$, for whose case $\Gamma_{1}=3.0924, \Gamma_{2}=1, \Gamma_{3}=-0.5$, and $\Gamma_{4}=0$. 


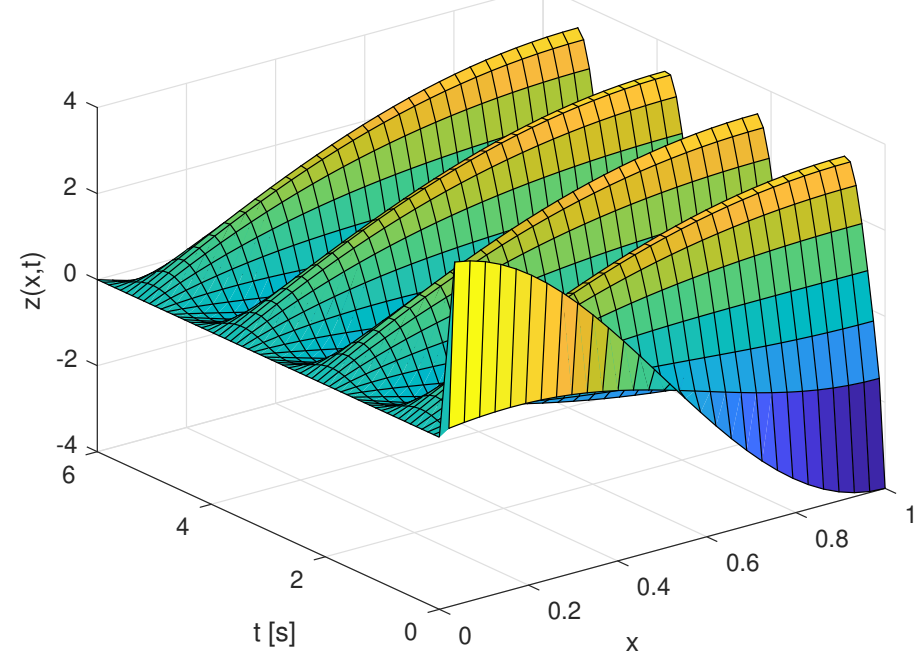

Figure 4. Plot of the solution surface for $\lambda=2.5$.

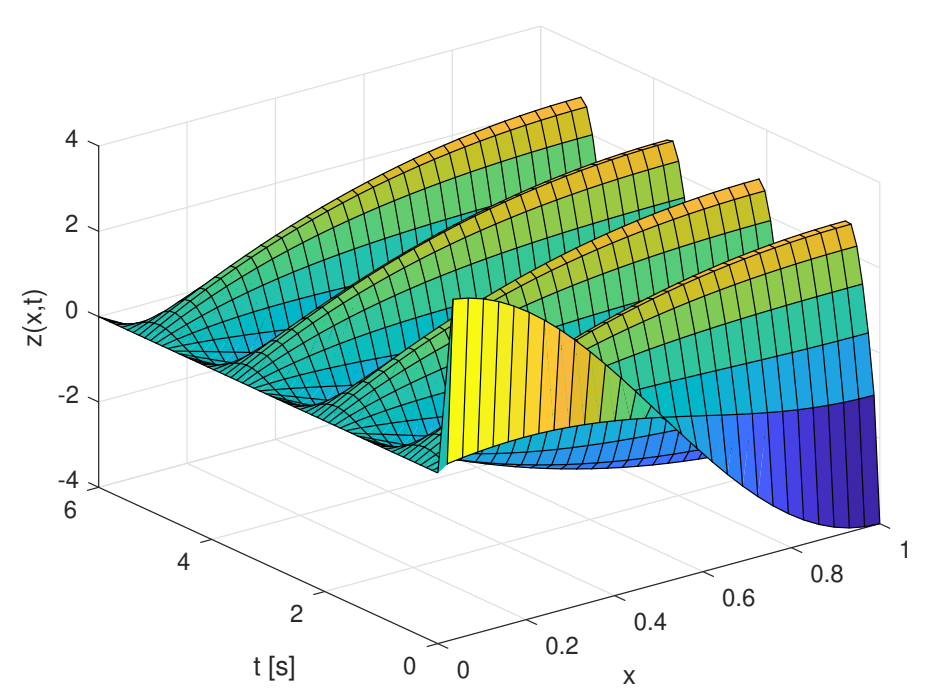

Figure 5. Solution surface for the case in which the reaction term is omitted, i.e., to the case for which $\lambda=0$.

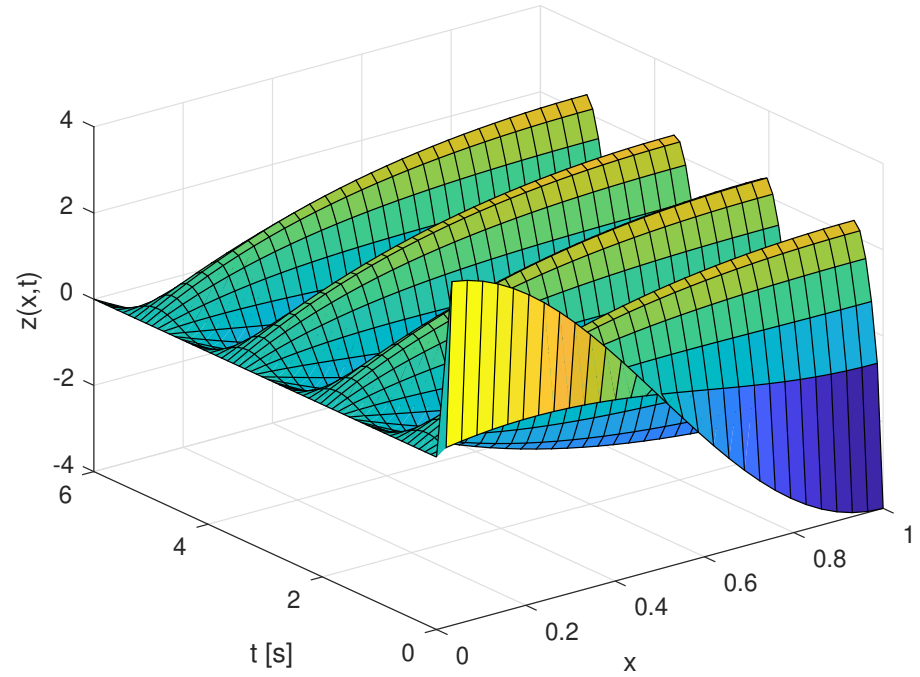

Figure 6. Plot of the solution surface for $\lambda=-2.5$. 


\section{Conclusions}

In [16], from an abstract control system model for the 1-D heat equation, the SFRP was solved in terms of the transfer function of the system, but with feedback control law in function of the state of the exosystem. The SFRP to the 1-D heat equation was solved for harmonic tracking and rejection of a constant disturbance. The SLDO (5) is present for most of the R-D equation types [34]. In our work, we propose the state operator for an abstract control system model to the 1-D R-D equation, in contrast with that in [16], namely, (13), as given in the form of the SLDO (12). Accordingly, the 1-D R-D equation is characterized, along the same lines as in [16], in terms of the Francis (regulator) equations, but with state operator (12) and then these are solved. The SFRP to the 1-D R-D equation is solved for harmonic tracking with harmonic disturbance rejection. The simulation results validate the performance of the regulator, i.e., the error tends to zero as time tends to infinity. From all of the above, we conclude that our proposal performs well, i.e., the SFRP for the 1-D R-D equation has solution. As future work, our proposal may be extended to those approaches in [16], and so on, on which the abstract control system model to the R-D equation could falls.

Author Contributions: Conceptualization, F.J.; methodology, F.J. and A.A.R.; software, A.A.R.; validation, F.J. and A.A.R.; formal analysis, F.J. and A.A.R.; investigation, F.J.; data curation, A.A.R.; writing-original draft preparation, F.J.; writing — review and editing, F.J.; visualization, A.A.R.; resources, F.J.; supervision, F.J.; funding acquisition, F.J.; project administration, F.J. All authors have read and agreed to the published version of the manuscript.

Funding: This research was funded by Tecnológico Nacional de México (TecNM) under project 8007.20-P and, partially, under grant number 30844 from EDD 2019 program.

Acknowledgments: This work was supported by CONACYT-SENER, México, through grant number 862135.

Conflicts of Interest: The authors declare no conflict of interest.

\section{Abbreviations}

The following abbreviations are used in this manuscript:

$\begin{array}{ll}\text { DPSs } & \text { Distributed Parameters Systems } \\ \text { EFRP } & \text { Error Feedback Regulation Problem } \\ \text { ODEs } & \text { Ordinary Differential Equations } \\ \text { PDEs } & \text { Partial Differential Equations } \\ \text { R-D } & \text { Reaction-Diffusion } \\ \text { SFRP } & \text { State Feedback Regulation Problem } \\ \text { SLBVP } & \text { Sturm-Liouville Boundary Value Problem } \\ \text { SLDO } & \text { Sturm-Liouville Differential Operator }\end{array}$

\section{References}

1. Morris, K. Control of Systems Governed by Partial Differential Equations in The Control Handbook, 2nd ed.; Levine, W.S., Ed.; CRC Press: Boca Raton, FL, USA, 2011.

2. Isidori, A. Nonlinear Control Systems; Springer: London, UK, 1995.

3. Isidori, A.; Byrnes, C.I. Output Regulation of Nonlinear Systems. IEEE Trans. Autom. Control 1990, 35, 131-140. [CrossRef]

4. Francis, B.A.; Wonham, W.M. The Internal Model Principle of Control Theory. Automatica 1976, 12, 457-465. [CrossRef]

5. Francis, B.A. The Linear Multivariable Regulator Problem. SIAM J. Control Optim. 1977, 15, 486-505. [CrossRef]

6. Wonham, W.M. Linear Multivariable Control: A Geometric Approach; Springer: New York, NY, USA, 1985.

7. Hautus, M.L.J. Linear Matrix Equations with Applications to the Regulator Problem. In Outils et modèles Mathématiques Pour L'automatique: L'analyse de Systèmes et le Traitement du Signal; Editions du Centre National de la Recherche Scientifique: Paris, France, 1982.

8. Knobloch, H.W.; Isidori, A.; Flockerzi, D. Topics in Control Theory; Birkhäuser Verlag: Basel, Switzerland, 1993. 
9. Pohjolainen, S.A. On The Asymptotic Regulation Problem for Distributed Parameter Systems. Control Distrib. Parameter Syst. 1983, 197-201. [CrossRef]

10. Pohjolainen, S.A. Robust Multivariable PI-Controller for Infinite Dimensional Systems. IEEE Trans. Autom. Control 1982, 27, 17-30. [CrossRef]

11. Schumacher, J.M. Dynamic Feedback in Finite- and Infinite-Dimensional Linear Systems; Mathematisch Centrum Tracts Centrum Voor Wiskunde en Informatica: Amsterdam, Netherlands, 1981; Volume 143.

12. Schumacher, J.M. Finite-Dimensional Regulators for a Class of Infinite-Dimensional Systems. Syst. Control Lett. 1983, 3, 7-12. [CrossRef]

13. Byrnes, C.I.; Laukó, I.G.; Gilliam, D.S.; Shubov, V.I. Output Regulation for Linear Distributed Parameter Systems. IEEE Trans. Autom. Control 2000, 45, 2236-2252.

14. Müller, P.H.; Kato, T. Perturbation theory for linear operators (Die Grundlehren der mathematischen Wissenschaften in Einzeldarstellungen mit besonderer Berücksichtigung der Anwendungsgebiete, Band 132) XX+592 S. m. 3 Fig. Springer-Verlag. Preis geb. DM 79, 20. Z. Angew. Math. Und Mech. 1967, 47. [CrossRef]

15. Curtain, R.F.; Zwart, H. An Introduction to Infinite-Dimensional Linear Systems Theory; Springer: New York, NY, USA, 1995.

16. Aulisa, E.; Gilliam, D. A Practical Guide to Geometric Regulation for Distributed Parameter Systems; CRC Press: Boca Raton, FL, USA, 2016.

17. Natarajan, V.; Gilliam, D.S.; Weiss, G. The State Feedback Regulator Problem for Regular Linear Systems. IEEE Trans. Autom. Control 2014, 59, 2708-2723. [CrossRef]

18. Xu, X.; Dubljevic, S. Output and Error Feedback Regulator Designs for Linear Infinite-Dimensional Systems. Automatica 2017, 83, 170-178. [CrossRef]

19. Deutscher, J. A Backstepping Approach to the Output Regulation of Boundary Controlled Parabolic PDEs. Automatica 2015, 57, 56-64. [CrossRef]

20. Deutscher, J. Finite-Time Output Regulation for Linear $2 \times 2$ Hyperbolic Systems using Backstepping. Automatica 2017, 75, 54-62. [CrossRef]

21. Zhou, H.C.; Guo, B.Z. Performance Output Tracking for One-Dimensional Wave Equation Subject to Unmatched General Disturbance and Non-Collocated Control. Eur. J. Control 2018, 39, 39-52. [CrossRef]

22. Humaloja, J.; Kurula, M.; Paunonen, L. Approximate Robust Output Regulation of Boundary Control Systems. IEEE Trans. Autom. Control 2019, 64, 2210-2223. [CrossRef]

23. Rebarber, R.; Weiss, G. Internal Model based Tracking and Disturbance Rejection for Stable Well-Posed Systems. Automatica 2003, 39, 1555-1569. [CrossRef]

24. Jin, F.F.; Guo, B.Z. Boundary Output Tracking for an Euler-Bernoulli Beam Equation with Unmatched Perturbations from a Known Exosystem. Automatica 2019, 109, 108507. [CrossRef]

25. Xu, X.; Dubljevic, S. Output Regulation for a Class of Linear Boundary Controlled First-Order Hyperbolic PIDE Systems. Automatica 2017, 85, 43-52. [CrossRef]

26. Logan, J.D. An Introduction to Nonlinear Partial Differential Equations; John Wiley \& Sons: Hoboken, NJ, USA, 2008.

27. Smoller, J. Shock Waves and Reaction-Diffusion Equations; Springer: New York, NY, USA, 1983.

28. Murray, J.D. Mathematical Biology I: An Introduction; Springer: Berlin/Heidelberg, Germany, 2002.

29. Murray, J.D. Mathematical Biology II: Spatial Models and Biomedical Applications; Springer: Berlin/Heidelberg, Germany, 2003.

30. Tveito, A.; Winther, R. Introduction to Partial Differential Equations A Computational Approach; Springer: Berlin/Heidelberg, Germany, 2005.

31. Banks, H.T.; Tran, H.T. Mathematical and Experimental Modeling of Physical and Biological Processes; CRC Press: Boca Raton, FL, USA, 2009.

32. Gahn, M.; Neuss-Radu, M.; Knabner, P. Homogenization of Reaction-Diffusion Processes in a Two-Component Porous Medium with Nonlinear Flux Conditions at the Interface. SIAM J. Appl. Math. 2016, 76, 1819-1843. [CrossRef]

33. Cardone, G.; Perugia, C.; Timofte, C. Homogenization Results for a Coupled System of Reaction-Diffusion Equations. Nonlinear Anal. 2019, 188, 236-264. [CrossRef]

34. Karafyllis, I.; Krstic, M. Input-to-State Stability for PDEs; Springer: Cham, Switzerland, 2019.

35. Krstic, M.; Smyshlyaev, A. Boundary Control of PDEs A Course on Backstepping Designs; SIAM: Philadelphia, PA, USA, 2008. 
36. Ramírez, A.A.; Jurado, F. Tracking Regulator Design with Disturbance Rejection to the Reaction-Diffusion Equation. In Proceedings of the 2019 16th International Conference on Electrical Engineering, Computing Science and Automatic Control (CCE), Mexico City, Mexico, 11-13 September 2019.

37. Seidman, T.I. Control of the Heat Equation in The Control Handbook, 2nd ed.; Levine, W.S., Ed.; CRC Press: Boca Raton, FL, USA, 2011.

38. Strauss, W.A. Partial Differential Equations an Introduction; John Wiley \& Sons: Hoboken, NJ, USA, 2008.

39. Mathews, J.; Walker, R.L. Mathematical Methods of Physics; The Benjamin/Cummings Publishing Co.: Menlo Park, CA, USA, 1970.

40. Boyce, W.E.; DiPrima, R.C.; Meade, D.B. Elementary Differential Equations and Boundary-Value Problems; John Wiley \& Sons: Hoboken, NJ, USA, 2017.

41. Kreyszig, E. Advanced Engineering Mathematics; John Wiley \& Sons: Hoboken, NJ, USA, 2006.

42. Farlow, S.J. Partial Differential Equations for Scientists and Engineers; Dover Publications: New York, NY, USA, 1993.

43. Holland, S.S., Jr. Applied Analysis by the Hilbert Space Method, an Introduction with Applications to the Wave, Heat, and Schrödinger Equations; Dover Publications: Mineola, NY, USA, 1990.

44. Kaplan, K. Advanced Calculus; Pearson Education: Boston, MA, USA, 2003.

45. Pazy, A. Semigroups of Linear Operators and Applications to Partial Differential Equations; Springer: New York, NY, USA, 1983.

Publisher's Note: MDPI stays neutral with regard to jurisdictional claims in published maps and institutional affiliations.

(C) 2020 by the authors. Licensee MDPI, Basel, Switzerland. This article is an open access article distributed under the terms and conditions of the Creative Commons Attribution (CC BY) license (http:/ / creativecommons.org/licenses/by/4.0/). 